\title{
Increasing the sensitivity of an optic level sensor with a wavelength and phase sensitive single-mode multimode single-mode (SMS) fiber structure
}

\author{
Omar Fuentes, Ignacio del Villar, Jose R. Vento, Abian B. Socorro, Elieser E. Gallego, Jesus M. \\ Corres, Member IEEE, Ignacio R. Matias Member, IEEE
}

\begin{abstract}
The sensitivity of a liquid level sensor based on a single-mode-multimode-single-mode (SMS) fiber structure has been increased by hydrofluoric acid etching. The etching process was analyzed and monitored both theoretical and experimentally, which permitted to observe that a sinusoidal spectrum can be obtained for low diameters. As an example, a 2.77 fold sensitivity increase was attained by etching from diameter 125 to $50 \mu \mathrm{m}$. Moreover, the sinusoidal shape of the optical spectrum permitted to monitor liquid level changes both in wavelength and in phase. The cross sensitivity of the sensor to refractive index and temperature was also studied.
\end{abstract}

Index Terms - optical fiber transducers, optical interferometry, phase measurement, SMS structure

\section{INTRODUCTION}

$\mathrm{A}$ level sensor is a device that measures the height of a liquid inside a tank, container or a similar recipient. In fact, many of these sensors can be found in nuclear facilities, bridges, swamps as well as naval ports, as examples of civil works where it is important to know the water level to avoid accidents [1][3]. Moreover, they are also present when monitoring the volume of liquids in hazardous applications such as fuel or oil storage [4].

Critical for the control of many processes in industrial applications, liquid level sensors can be classified into two main types: point level sensors and continuous level sensors. The first type is used as an alarm, indicating that the threshold level has been overcome [5]. On the other hand, a continuous type sensor is more complex and can track the liquid level in an industrial system, measuring it within a previously defined range [6]. The output of these measuring systems is an analog signal directly related to the liquid level inside the container, which can be taken into account to manage the control of the different processes within the facilities.

Manuscript received on May 16, 2017. This work was supported by the Spanish Agencia Estatal de Investigación (AEI) and Fondo Europeo de Desarrollo Regional (FEDER) (TEC2016-78047-R) and by Pinar del Río University.

Omar Fuentes, Jose R. Vento and Elieser E. Gallego are with the Department of Telecommunications and Electronics of Pinar del Río University, Cuba (emails: omarf@upr.edu.cu,vento@upr.edu.cu, elieser@upr.edu.cu).
Among the different ways to address the design of liquid level sensors, capacitive, ultrasonic and optical strategies are the main options to develop [7-9], since they guarantee simple, easy-to-handle and cost-effective devices. From an optical point of view, the issue addressed in this contribution, a simple setup can be considered, consisting of a light source and a photodetector sending and receiving light to/from a container wall. The reflected power is detected and processed to calculate the liquid level, something similar to radar or ultrasonic devices [9]. This is an example of an optical wireless technology with good commercial acceptation.

This work will show a spectrum-based technique, which monitors wavelength changes due to surrounding medium refractive index changes. This ensures high sensitivity and resolution measurements, as it will be shown during the article.

In this sense, a wired technology is proposed here, which consists of tracking the light variations inside an optical fiberbased structure. Fiber-optic-based devices present interesting properties such as immunity against electromagnetic interferences, possibility of multiplexing of sensors and small size, combined with the fact of being resistant to hazardous or inflammable media, which is interesting in order to make this technology versatile in many applications [10], [11]. The most recent optical approaches to liquid level sensors suggest an indirect measurement of the liquid level by studying the effect of pressure in the device [12], or a direct measurement by analyzing the wavelength shift when part of the device is introduced in liquid (long-period fiber gratings and interferometers can be used [13,14]). The latter case is considered in this manuscript: a multimodal interferometer (MMI) based on a single-mode - multimode - single-mode optical fiber structure (SMS) is used.

The SMS structure has turned out to be a cost-effective structure, with many ways to be optimized and very easy to

Ignacio Del Villar, Abian B. Socorro, Jesus M. Corres and Ignacio R. Matias are with the Institute of Smart Cities and the Electrical and Electronic Engineering Department of the Public University of Navarre, 31006 Pamplona, Navarre, Spain (e-mails: ignacio.delvillar@unavarra.es, ab.socorro@unavarra.es,jmcorres@unavarra.es,natxo@unavarra.es). 
fabricate and characterize, as it can be found elsewhere [15], [16]. A recent work by Antonio-Lopez et al. reports the measurement of liquid level based on an SMS structure that acts as a continuous level sensor [17]. The novelty presented in our contribution is the fact of getting the best performance of the SMS structure in terms of sensitivity and resolution, based on an etching process that improves the behavior of the original SMS-based waveguide.

In addition to this, a novel measurement method based on Fast Fourier Transform (FFT) will be presented, which uses the phase shift as a technique for detecting liquid level changes.

\section{EXPERIMENTAL SECTION}

The experimental setup for the level sensor based on SMS fiber is depicted in Fig. 1. The SMS structure was obtained by splicing a segment of coreless multimode fiber (MMF) from POFC Inc. to two standard SMF pigtails from Telnet Redes Inteligentes Inc. The diameter of both the SMF and MMF segments was $125 \mu \mathrm{m}$.

One end on the SMS structure was connected to an Agilent 83437A white light source and the other end was connected to an HP 86142A optical spectrum analyzer (OSA). The liquid level was detected by progressively immersing the sensor in the liquid with a programmed robot sequence. The output spectrum of the sensor was continuously monitored for each liquid level and characterized at different liquid temperatures by using a heater and an HP 34970 temperature recorder. In addition to this, the sensor was also characterized with different glycerol in water solutions ( $20 \%$ and $40 \%$ ), which permitted to analyze the cross-sensitivity of the sensor to refractive index changes.

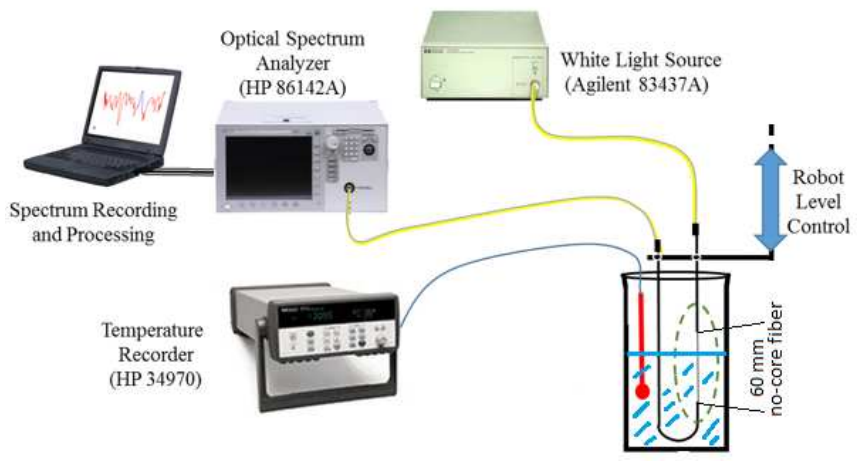

Fig. 1. Experimental setup

\section{ChaRACTERIZATION OF SMS FIBER}

As it was indicated in the introduction, SMS fibers can be tuned to work in two modes of operation. The first one is the self image effect (a light field condensation between the several guided modes in a particular plane $[15,18]$ ), which permits to obtain a maximum in the transmission spectrum that is distinguished from other maxima obtained by a fractional constructive interference.

The wavelength where this maximum is located can be obtained with analytical expression (1), which permits to obtain the MMF section length adequate for setting the fourth selfimage band at a specific wavelength $[17,19]$ :

$$
\lambda=\frac{4 D^{2} n}{Z}
$$

where $D$ is the diameter of the MMF section, $n$ is the refractive index of the silica [20], material used to manufacture the optical fiber), $\mathrm{Z}$ is the MMF segment length and $\lambda$ is the operational wavelength.

The setup depicted in Fig. 1 permits to monitor the optical spectrum from 1150 to $1680 \mathrm{~nm}$. For this reason, a MMF length $60 \mathrm{~mm}$ was selected, which according to expression (1) leads to the generation of the self-image band at $1504.16 \mathrm{~nm}$.

In Fig. 2 the transmission spectrum for this design is shown. For the sake of comparison we also present the numerical spectrum obtained with FIMMAVE ${ }^{\circledR}$. The propagation was calculated with FIMMPROP, a module integrated with FIMMWAVE. For both SMF and MMF sections, the finite difference method FDM was used, because it is the most accurate method available for a cylindrical waveguide. For the SMF sections one mode was analyzed, whereas 30 modes were analyzed in the case of the MMF section, a number that permitted to achieve a convergence in the results.
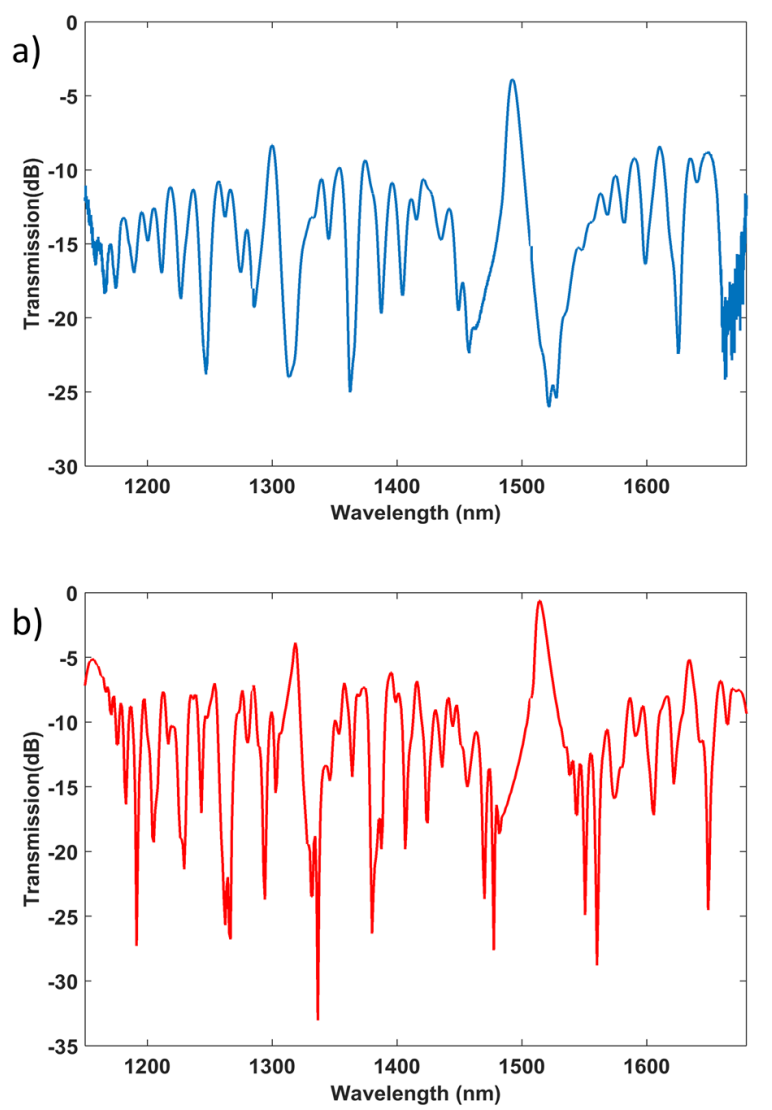

Fig. 2. Transmission spectrum for an SMS fiber with diameter $125 \mu \mathrm{m}$ and MMF length $60 \mathrm{~mm}$ : (a) Experimental; (b) Simulation with FIMMWAVE.

The self-image band can be observed both in the experimental and in the theoretical plot at approximately 1500 $\mathrm{nm}$, which agrees with the result obtained evaluating expression (1): $1504.17 \mathrm{~nm}$. The not perfect match between the position of 
the self-image band in the experimental results and in the simulation is due to $\pm 0.5 \mathrm{~mm}$ error in the length of the MMF segment. As an example, if the MMF segment is $60.5 \mathrm{~mm}$, the self-image is located at $1491.73 \mathrm{~nm}$ according to expression (1).

The key parameter that leads to a sensitivity enhancement to the surrounding medium refractive index in an SMS structure is the diameter [16], and the liquid level sensor developed in next section is also based on the sensitivity to refractive index: if the sensor is immersed completely in water the transmission and attenuation bands in the optical spectrum will experience a wavelength shift due to the change of refractive index of the air (1) to that of the water (1.333). Between this two extreme cases the attenuation and transmission bands will adopt different positions depending of the section of the MMF segment that is immersed in water. And the reduction of the fiber diameter will lead to an increase of the wavelength range swept between the two extreme cases (no liquid and sensor completely immersed in liquid). Consequently, the best option will be to reduce the fiber diameter with an etching process (see Fig. 3). As an example of the smoothness of the etching process, an image of the fiber after etching is presented in Fig. 3(c).

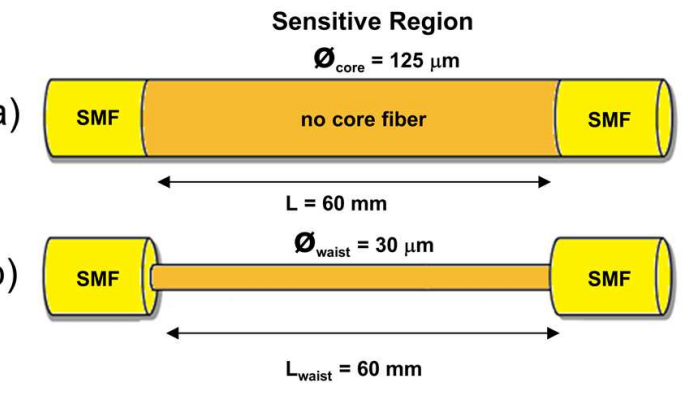

C)

Fig. 3. SMS structure: (a) before etching; (b) after etching; (c) microscope photograph of the no core section of the sensor after etching.

In Fig. 4 the experimental transmission spectra obtained are mapped as a function of both the wavelength and time for the SMS structure under study during the etching process. For the sake of comparison we include also the numerical results for a diameter of up to $30 \mu \mathrm{m}$. The SRI value outside the fiber is 1.22 , which permits to simulate that the fiber is immersed in HF. A video of the etching process is presented also in Visualization 1 (experimental results) and Visualization 2 (numerical results), showing the spectrum evolution as a function of the etching time.

Both visualizations show that as well as the waveguide diameter is reduced it gets to a point in which the spectrum presents a sinusoidal shape. This is the second mode of operation of the SMS structure and it permits to use the device as both a wavelength and a phase sensitive device, as it will be shown later. In Fig. 5 an experimental spectrum after 44 minutes and a numerical spectrum obtained with a diameter of $30 \mu \mathrm{m}$ are depicted.
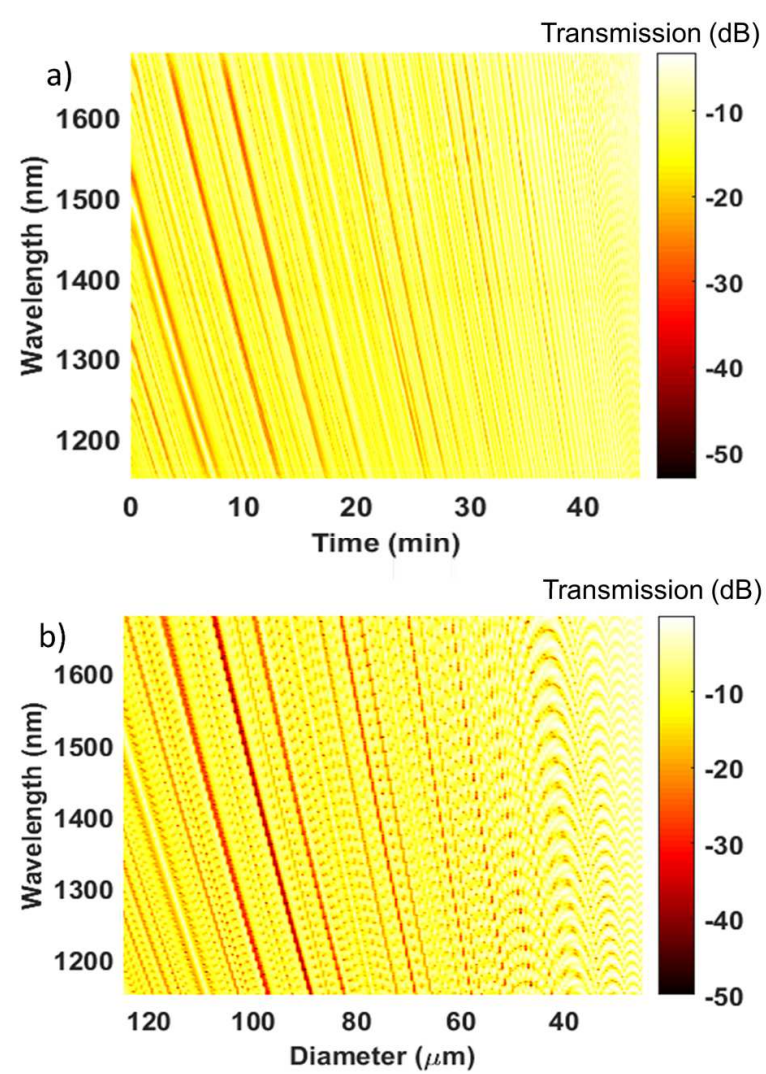

Fig. 4. Spectral evolution (transmission in $\mathrm{dB}$ ) when decreasing the SMS fiber diameter: (a) Experimental results; (b) Numerical results. In Visualization 1 and Visualization 2 the etching process up to $30 \mu \mathrm{m}$ SMS are shown interactively.
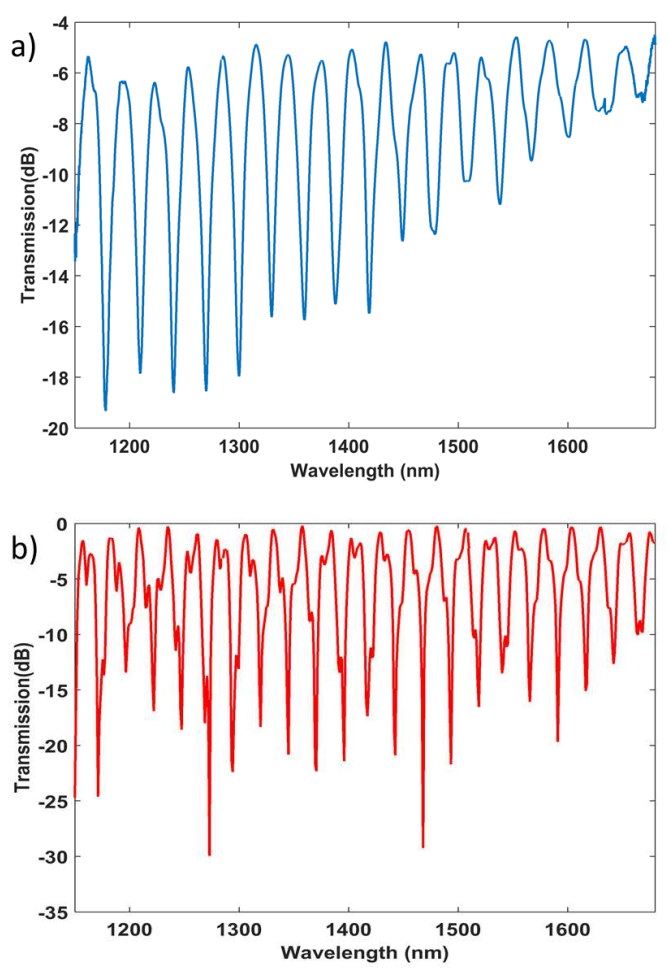

Fig. 5. Transmission spectrum for an SMS fiber with MMF length $60 \mathrm{~mm}$ after etching: (a) Experimental results after 44 minutes of etching; (b) Simulation with FIMMWAVE for a diameter $30 \mu \mathrm{m}$. 


\section{LEVEL SENSOR RESULTS}

Since the SMS fiber is an interferometer, the progressive immersion of the device in a liquid will lead to a wavelength shift of the optical spectrum [17]. For the sake of comparison, and once the performance of the sensor during etching is known, a new sensor with the same characteristics will be analyzed both before etching (in self-image band mode) and after etching (in sinusoidal spectrum mode).

\section{A. Self-image band mode}

Before etching, the sensor was immersed in the liquid in steps of $10 \mathrm{~mm}$. Fig. 6(a) shows the wavelength shift of the optical spectrum as a function of the liquid level in millimeters, whereas in Fig. 6(b) the central wavelength of the self-image band has been tracked during the immersion process as a function of time. The sensitivity of the device is $0.15 \mathrm{~nm} / \mathrm{mm}$, which is very similar to the $0.17 \mathrm{~nm} / \mathrm{mm}$ attained in [17].

It is not possible to explain the wavelength shift to the red with expression (1) because the surrounding medium refractive index is not considered in it (it is an approximation). However, the wavelength shift to the red can be understood if we consider that the effective index of modes guided in the MMF section is modified by the external medium [16]. In the level sensor the surrounding medium refractive index increases when the device is immersed in liquid and, similarly to the phenomenon observed in [20], the difference between the effective indices of modes guided in the fiber increases, something that can be observed in Fig. 3 of [21]. Focusing on constructive interference, this phenomenon occurs if the difference between the phases of modes is a multiple of $2 \pi$. The phase is directly proportional to the separation between the effective indices of the modes guided in the MMF section and to the length of the MMF section, and inversely proportional to the incident wavelength. In view of the fact that the difference in phase is inversely proportional to the incidence wavelength, this last parameter must be increased in order to continue satisfying the condition for constructive interference. In other words, there is a wavelength shift to the red, which also occurs for bands with destructive interference, following the same explanation but for a phase difference of an uneven multiple of $\pi$.
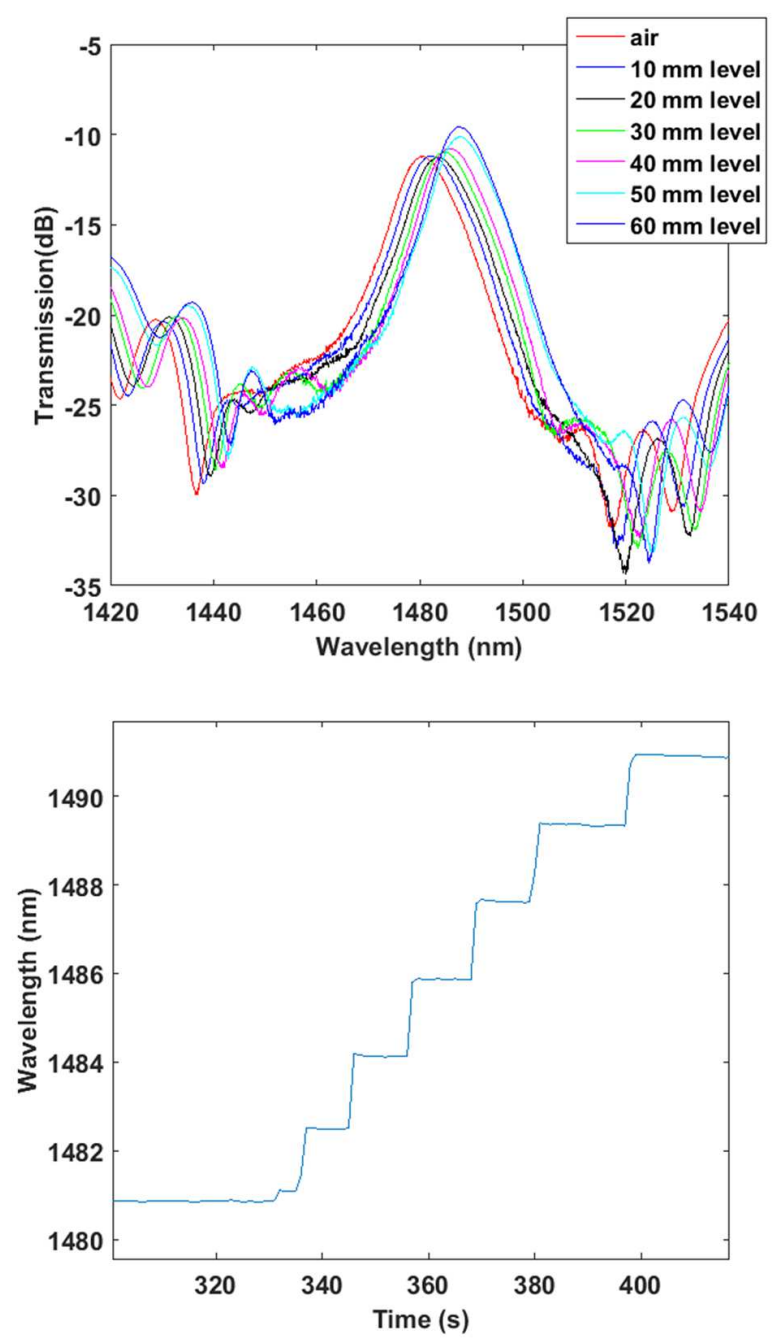

Fig. 6. Controlled liquid level sensor based on an unetched SMS fiber with diameter $125 \mu \mathrm{m}$ and length $60 \mathrm{~mm}$. (a) Transmission spectra for different liquid levels (b) Wavelength shift of the self-image band as a function of time

\section{B. Sinusoidal spectrum mode}

After characterizing the self-image band as a function of the refractive index, the device was immersed in hydrofluoric acid (HF) up to the moment when a sinusoidal spectrum could be monitored (35 minutes). The diameter of the SMS structure after etching was $50 \mu \mathrm{m}$. For the sake of comparison, a dip located at $1490 \mathrm{~nm}$, a similar wavelength to the self-image peak at $1481 \mathrm{~nm}$ in Fig. 6(a), was chosen. The reason for selecting a dip instead of a peak after etching was that the dip was narrower that the peak, which is important in terms of accuracy in the determination of the wavelength shift as a function of the liquid level. 

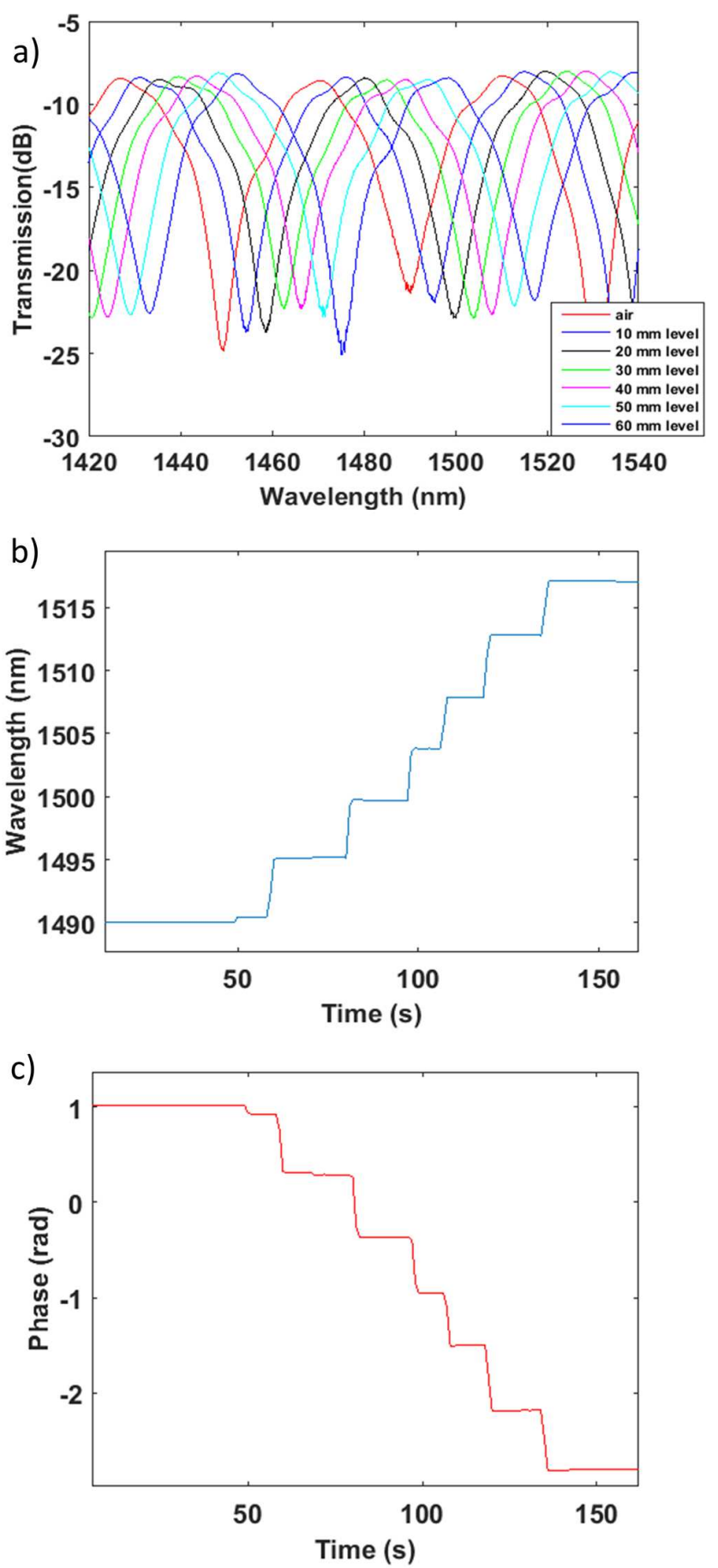

Fig. 7. Controlled liquid level sensor based on an etched SMS fiber with diameter $50 \mu \mathrm{m}$ and length $60 \mathrm{~mm}$. (a) Transmission spectra for different liquid levels; (b) Wavelength shift of one of the bands as a function of time; (c) Phase shift of the fundamental frequency as a function of time

The sensor was analyzed in the same way after the etching (see Fig. 7). In this case the wavelength shift was 0.41 $\mathrm{nm} / \mathrm{mm}$.

It is remarkable to mention that the ratio between the initial and the final diameter after etching (from $125 \mu \mathrm{m}$ to $50 \mu \mathrm{m}$ ) is 2.5 , whereas the ratio between the sensitivity after and before etching was 2.77 (sensitivity increases from $0.15 \mathrm{~nm} / \mathrm{mm}$ to
$0.41 \mathrm{~nm} / \mathrm{mm}$ ). This fact agrees with a sensitivity increase proportional to the diameter reduction [18]. Moreover, in view of the sinusoidal shape of the optical spectrum obtained with the etched SMS structure, and according to what was explained in previous section, it was possible to analyze both the wavelength shift and the phase shift as a function of time (Fig. 7(b) and Fig. 7(c)). Regarding the phase shift, a sensitivity of $0.0615 \mathrm{rad} / \mathrm{mm}$ was attained. This proves the ability of this sensor to be monitored with a phase interrogator, which is interesting in terms of reducing costs.

This phase shift tracking was possible because the Fast Fourier Transform FFT corresponding to the sensor after the etching presents a different shape if compared to the FFT before etching. In Fig. 8(b), the fundamental frequency corresponding to a period of the spectra in Fig. 7(a) can be observed. However, this is not the case in Fig. 8(a). In this case, the fundamental mode of one of the spectra in Fig. 6(a) cannot be appreciated. This is because the spectrum is not sinusoidal.
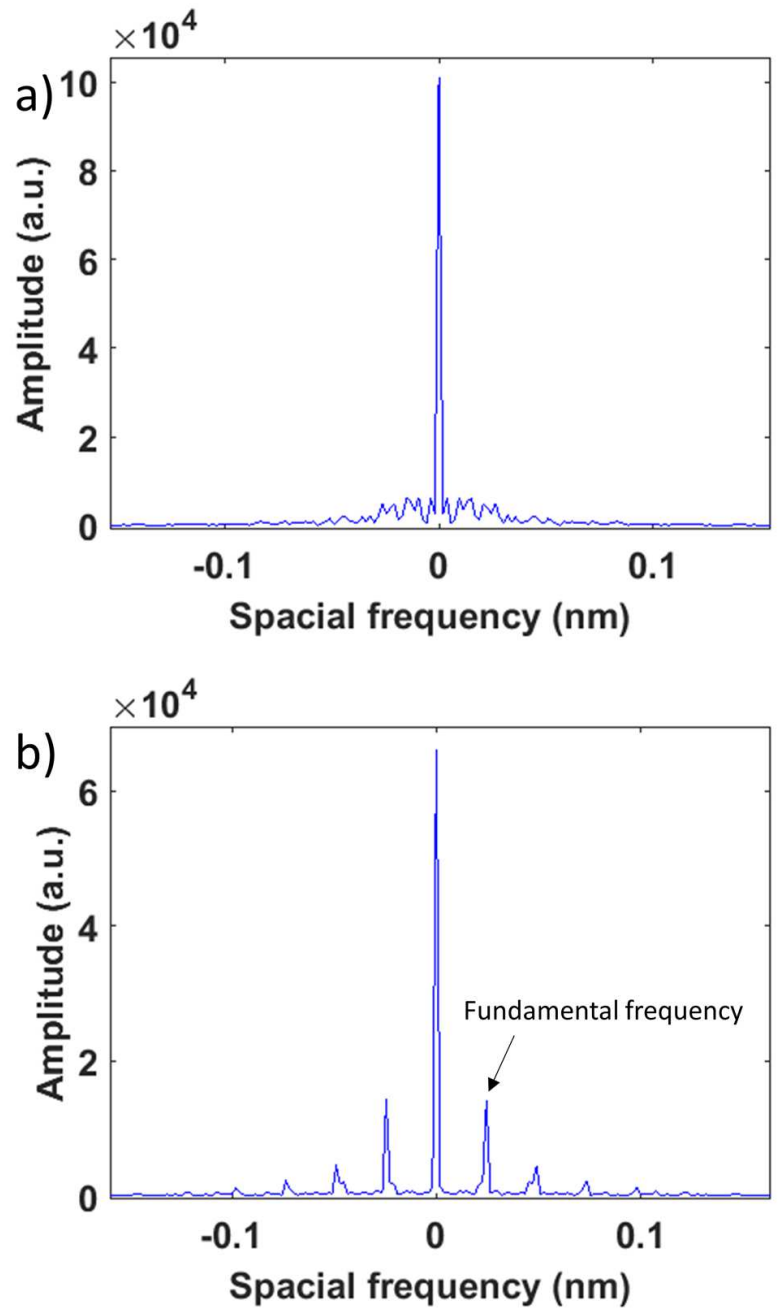

Fig. 8. Amplitude of the Fast Fourier Transform: (a) before etching; (b) after etching.

In addition to the previous results, a more accurate analysis in steps of $5 \mathrm{~mm}$ was performed for the sensor after etching (Fig. 
9(a), (b)). Moreover, the sensor was analyzed both during the immersion and extraction from the liquid, and it recovers its initial state, as it can be observed.

It is important to mention that, with an MMF segment of higher length, the period of the sinusoidal spectrum could be reduced to such a point that the phase shift exceeds the period of the signal. However, in view that the increase or decrease of the liquid is progressive, algorithms for detecting discontinuities that will detect when a phase shift higher than $2 \pi$ is attained could correct this problem. Another problem in increasing the device length would be an increase of losses in the transmission spectrum.

Finally, the central wavelength shift as a function of the liquid level of both the sensor before and after etching is compared in Fig 10(a). Fig. 10(b) shows the wavelength shift as a function of time in steps of $1 \mathrm{~mm}$ for the sensor after etching.
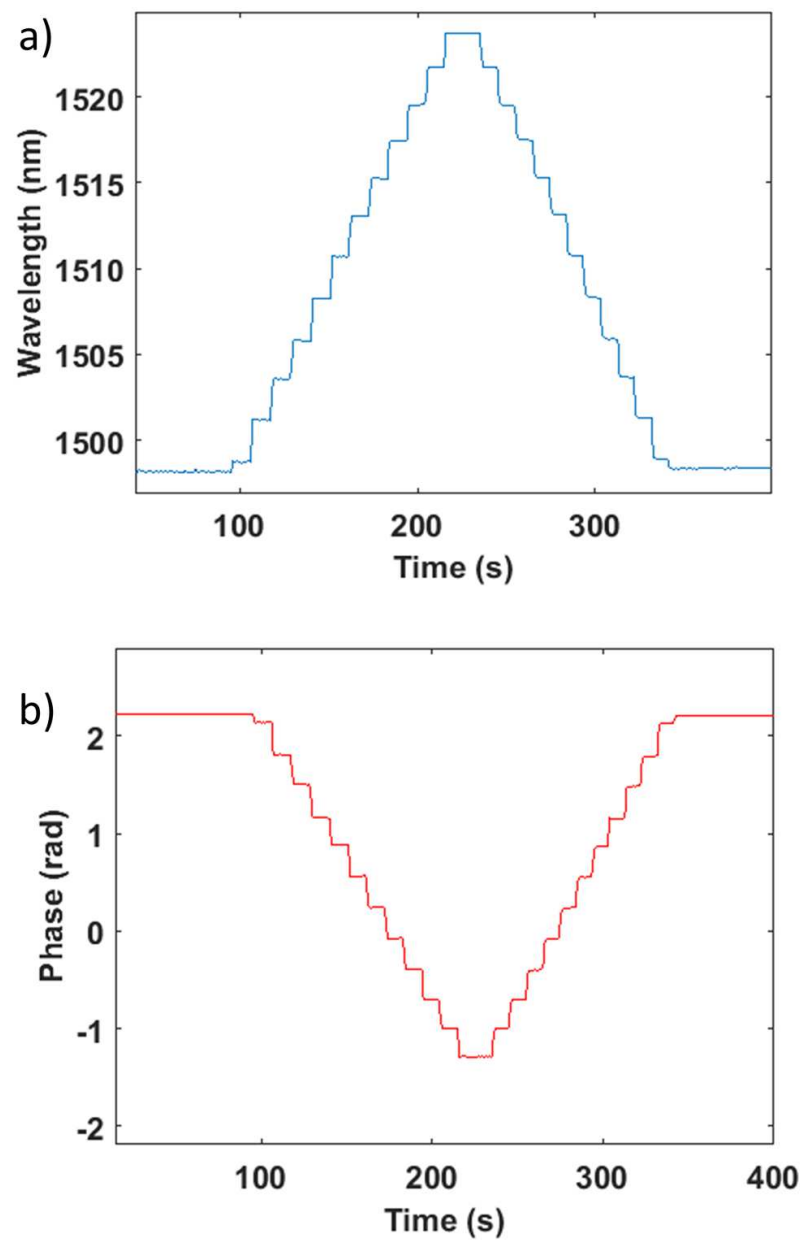

Fig. 9. Controlled measurements for liquid level sensor based on an etched SMS fiber with diameter $50 \mu \mathrm{m}$ and length $60 \mathrm{~mm}$. (a) Wavelength shift of one of the bands as a function of time; (b) Phase shift of the fundamental frequency as a function of time.
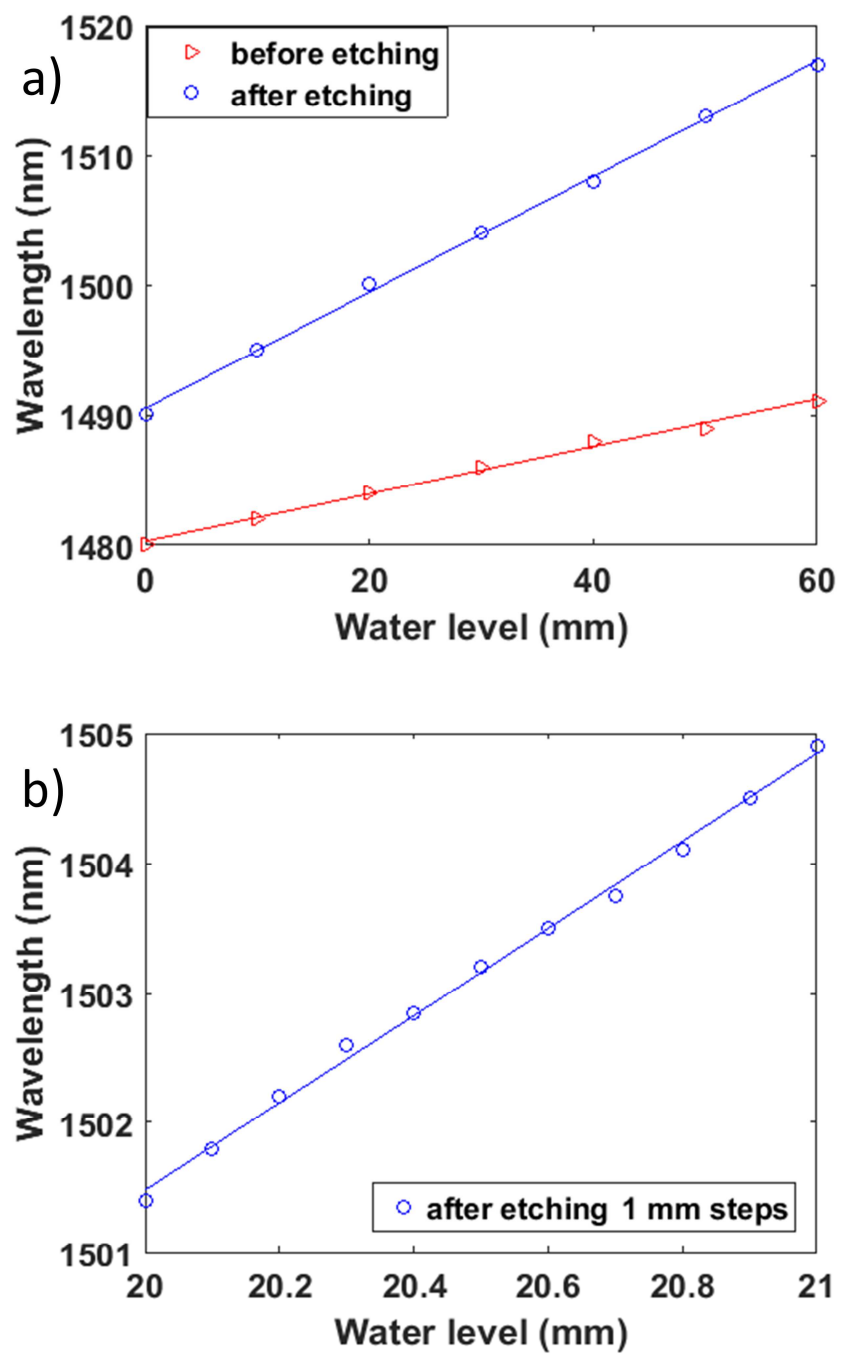

Fig. 10. (a) Wavelength shift of $60 \mathrm{~mm}$ length SMS sensor before and after etching; (b) Wavelength shift for the SMS sensor after etching in steps of $1 \mathrm{~mm}$.

\section{Cross sensitivity}

The cross sensitivity to temperature and refractive index of the SMS sensor after etching was analyzed in Fig. 11 and Fig. 12. For the sake of simplicity, only the wavelength shift is analyzed. Nonetheless, the performance as a function of phase would be similar.

In Fig. 11, the water temperature was increased in steps of $10{ }^{\circ} \mathrm{C}$. The slope of the plots as a function the liquid level was constant and the sensitivity to temperature was $36 \mathrm{pm} /{ }^{\circ} \mathrm{C}$. This constant performance, independent of the liquid level, indicates that the sensitivity is mainly due to the expansion of the sensor. In this sense, it is important to indicate that the sensor was glued to a polylactic acid holder, with a higher expansion coefficient of $80 \times 10^{-6} /{ }^{\circ} \mathrm{C}$ (higher than silica), which suggests that with a material with a lower expansion coefficient the cross-sensitivity to temperature could be reduced.

Regarding the refractive index cross sensitivity, the sensor was immersed in water and different glycerol in water solutions 
(20\% and $40 \%$ respectively). These solutions correspond with refractive indices $1.321,1.352$ and 1.382 in the wavelength range analyzed [22], as shown in Fig. 12. In view that the maximum variation from water to glycerol $40 \%$ is $21 \mathrm{~nm}$, it can be said that the cross-sensitivity to refractive index in the refractive index range $1.321-1.382$ is $344.2 \mathrm{~nm} /$ refractive index unit (nm/RIU).

This sensitivity indicates the possibility that water refractive index variation as a function of temperature could play a role in the wavelength shift observed in Fig. 11 as a function of temperature. However, the refractive index of water decreases as the temperature increases [23], which should lead to a wavelength shift to the blue as a function of temperature, contrary to the effect observed in Fig. 11. Consequently, the water refractive index variation is not the main effect in Fig. 11. Moreover, by selecting a different material for the plastic holder that maintains the fiber straight it could be possible to counterbalance both effects and to reduce the sensitivity to temperature.

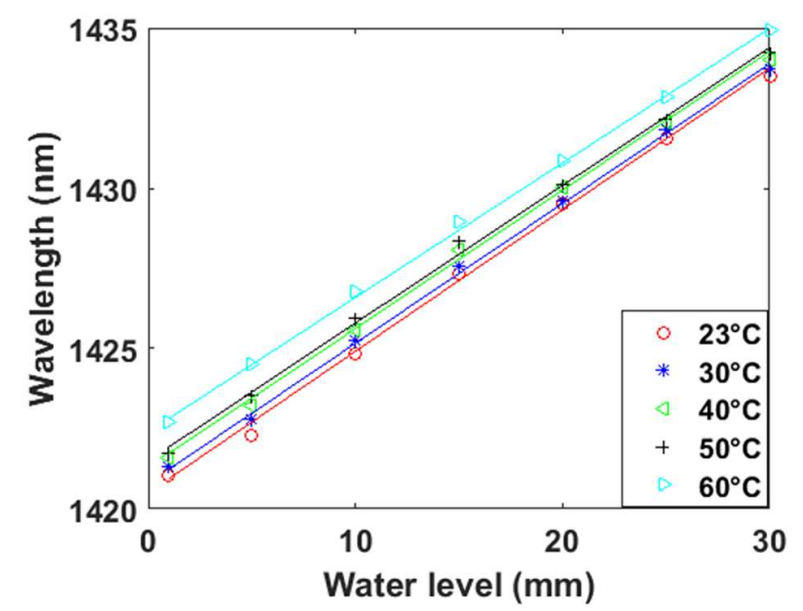

Fig. 11. Wavelength shift of $60 \mathrm{~mm}$ length SMS sensor after etching at different temperatures (Average sensitivity $=36 \mathrm{pm} /{ }^{\circ} \mathrm{C}$ ).

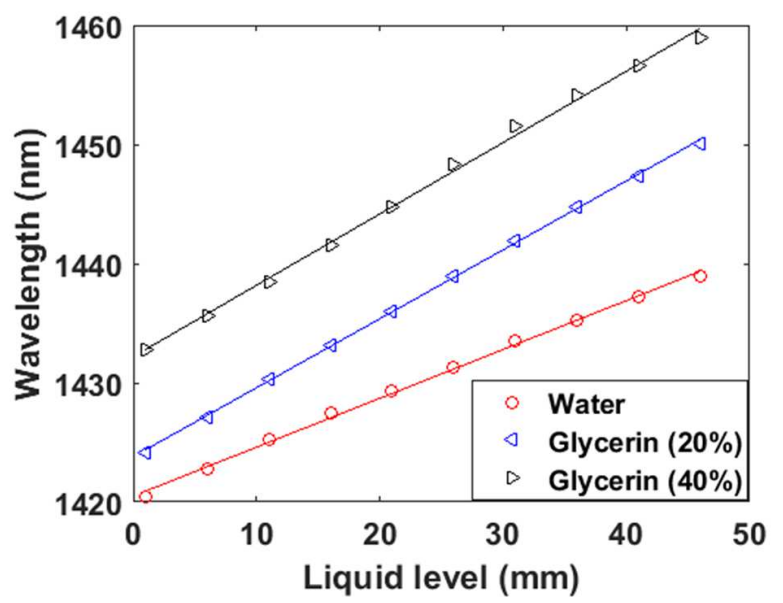

Fig. 12. Wavelength shift of $60 \mathrm{~mm}$ length SMS sensor after etching immersed in different liquids.

\section{CONCLUSIONS}

By monitoring the etching of an SMS structure it has been possible to observe two modes of operation in the optical spectrum. The first one is the self-image band, whereas the second one is a sinusoidal spectrum. This last mode of operation is obtained after etching. Consequently, two advantages are obtained at the same time: a sensitivity increase thanks to the diameter reduction and the possibility to both track in wavelength and phase the sensor. As an example of the performance for the second mode of operation, a sensor etched up to a diameter of $50 \mu \mathrm{m}$ has been analyzed. The sensitivity is improved by a factor of 2.77 , what involves the same factor of the diameter reduction. This suggests the possibility of obtaining more sensitive devices by gently reducing its diameter. In addition to this, it has been proved that the phase shift of the fundamental frequency follows the same tendency than the wavelength shift when the sensor is immersed in liquid. Consequently, the path is open towards a cost effective device where an interrogator instead of an optical spectrum analyzer is used.

\section{REFERENCES}

[1] K. Loizou and E. Koutroulis, "Water level sensing: State of the art review and performance evaluation of a low-cost measurement system," Measurement, vol. 89, pp. 204-214, 2016.

[2] Y. Sang and X. Li, "Novel characterization of the large water discharge," Instrumentation Science and Technology, vol. 45, no. 4, pp. 349-365, 2017.

[3] F. Bandini, J. Jakobsen, D. Olesen, J. A. Reyna-Gutierrez, and P. BauerGottwein, "Measuring water level in rivers and lakes from lightweight Unmanned Aerial Vehicles," Journal of Hydrology, vol. 548, pp. 237250, 2017.

[4] D. Y. Song, D. C. Lv, and Z. Q. Liu, "Design of Oil-Water Interface Meter Based on Segmental Capacitance Sensors," Applied Mechanics and Materials, vol. 475-476, pp. 132-135, 2013.

[5] W. Tang, P. Zhang, Y. Liu, L. H. Wang, and L. F. Liu, "Shiplock MultiChannel Water Level Monitoring and Message Interaction," Applied Mechanics and Materials, vol. 321-324, pp. 586-591, 2013.

[6] A. Pozo, F. Pérez-Ocón, and O. Rabaza, "A Continuous Liquid-Level Sensor for Fuel Tanks Based on Surface Plasmon Resonance," Sensors, vol. 16, no. 5, p. 724, May 2016.

[7] B. Kumar, G. Rajita, and N. Mandal, "A Review on Capacitive-Type Sensor for Measurement of Height of Liquid Level," Measurement and Control, vol. 47, no. 7, pp. 219-224, Sep. 2014.

[8] B. Zhang, Y.-J. Wei, W.-Y. Liu, Y.-J. Zhang, Z. Yao, L.-H. Zhao, and J.J. Xiong, "A Liquid Level Measurement Technique Outside a Sealed Metal Container Based on Ultrasonic Impedance and Echo Energy," Sensors, vol. 17, no. 1, p. 185, 2017

[9] H. K. Singh, S. K. Chakroborty, H. Talukdar, N. M. Singh, and T. Bezboruah, "A New Non-Intrusive optical technique to measure transparent liquid level and volume," IEEE Sensors Journal, vol. 11, no. 2, pp. 391-398, 2011.

[10] A. A. Kazemi, C. Yang, and S. Chen, "Fiber optic liquid level sensor system for aerospace applications," vol. 9202, p. 920202, 2014.

[11] W. Yoo, H. Sim, S. Shin, K. Jang, S. Cho, J. Moon, and B. Lee, “A FiberOptic Sensor Using an Aqueous Solution of Sodium Chloride to Measure Temperature and Water Level Simultaneously," Sensors, vol. 14, no. 10, pp. 18823-18836, 2014.

[12] D. Sengupta and P. Kishore, "Continuous liquid level monitoring sensor system using fiber Bragg grating," Optical Engineering, vol. 53, no. 1, p. 17102, 2014.

[13] S. Khaliq, S. W. James, and R. P. Tatam, "Fiber-optic liquid-level sensor using a long-period grating," Optics Letters, vol. 26, no. 16, pp. 12241226, 2001.

[14] X. Liu, X. Zhang, Y. Liu, Z. Liu, and W. Peng, "Multi-point fiber-optic refractive index sensor by using coreless fibers," Optics Communications, vol. 365, pp. 168-172, 2016. 
[15] S. Silva, E. G. P. Pachon, M. a. R. Franco, J. G. Hayashi, F. X. Malcata, O. Frazão, P. Jorge, and C. M. B. Cordeiro, "Ultrahigh-sensitivity temperature fiber sensor based on multimode interference," Applied Optics, vol. 51, no. 16, p. 3236, 2012.

[16] I. Del Villar, A. B. Socorro, J. M. Corres, F. J. Arregui, I. R. Matias, and S. Member, "Optimization of Sensors Based on Multimode Interference in Single-Mode - Multimode - Single-Mode Structure," Journal of Lightwave Technology, vol. 31, no. 22, pp. 3460-3468, 2013.

[17] J. E. Antonio-Lopez, J. J. Sanchez-Mondragon, P. Likamwa, and D. a May-Arrioja, "Fiber-optic sensor for liquid level measurement," Optics letters, vol. 36, no. 17, pp. 3425-3427, 2011.

[18] I. Del Villar, A. B. Socorro, J. M. Corres, F. J. Arregui, and I. R. Matias, "Refractometric sensors based on multimode interference in a thin-film coated single-mode-multimode-single-mode structure with reflection configuration," Applied optics, vol. 53, no. 18, pp. 3913-3919, 2014.

[19] L. B. Soldano, E. C. M. Pennings, "Optical Multi-Mode Interference Devices Based on Self-Imaging: Principles and Applications," J. Lightwave Technol. vol. 13, no. 4, pp. 615-627, 1995.

[20] A. B. Socorro, I. Del Villar, J. M. Corres, F. J. Arregui, and I. R. Matias "Mode transition in complex refractive index coated single-modemultimode-single-mode structure," Opt. Express, vol. 21, no. 10, pp 12668-12682, 2013

[21] I. Del Villar, A. B. Socorro, J. M. Corres, F. J. Arregui, and I. R. Matias, "Sensitivity optimization with cladding-etched long period fiber gratings at the dispersion turning point," Opt. Express, vol. 24, no. 16, pp. 17680$17685,2016$.

[22] I. H. Malitson, "Interspecimen comparison of the refractive index of fused silica," J. Opt. Soc. Am., vol. 55, pp. 1205-1209, 1965.

[23] A. N. Bashkatov and E. A. Genina. "Water refractive index in dependence on temperature and wavelength: a simple approximation". Proc. SPIE 5068, Saratov Fall Meeting 2002: Optical Technologies in Biophysics and Medicine IV, 393, 2003.

Omar Fuentes is a Automatic Engineer (1996) from Higher Polytechnic Institute "José A. Echeverria (CUJAE), La Habana, Cuba". Currently he works as Assistant Professor in the Department of Telecommunication and Electronic Engineering in Pinar del Río University (UPR).

Ignacio Del Villar received his MS degree in Electrical and Electronic Engineering and his Ph.D. degree, specialty in Optical Fiber Sensors, in 2002 and 2006, respectively, from the Public University of Navarra (UPNA). During 2004 was a visiting scientist at the Institute d'Optique (Orsay, France) and in 2005 he was a visiting scientist at the Applied Physics Department of the University of Valencia (Burjassot, Spain). $\mathrm{He}$ is presently a Reader at the Public University of Navarra since 2008, an Associate Editor of the Optics \& Laser Technology Journal since 2012. His research interest includes optical fiber sensors and the effect of nanostructured coatings deposited on waveguides, where he has co-authored more than 100 chapter books, journals and conference papers

José R. Vento is a Telecommunication Engineer (1986) from Higher Polytechnic Institute "José A. Echeverria (CUJAE), La Habana, Cuba" and his Ph.D. degree in the Polytechnic University of Madrid (UPM), Madrid, Spain, in 1996, respectively. Currently he works as Assistant Professor in the Department of Telecommunication and Electronic Engineering in Pinar del Río University (UPR).

Abian B. Socorro is an Electrical and Electronic Engineer (2010), M.Sc. in Biomedical Engineering (2012) and PhD in Engineering (2015) by the Public University of Navarre (PUNUPNA), Pamplona, Navarre, Spain. $\mathrm{He}$ was at the Armani
Research Lab (University of Southern California, Los Angeles, CA, USA) as a visiting PhD student in 2014. He is currently researching with a post-doctoral fellowship at UPNA towards the design and development of optical fiber-based biosensors. His research interests include fiber-optic (bio) sensors, fiber in the body, surface biofunctionalization and other biomedical engineering applications.

Elieser E. Gallego is a Telecommunication and Electronic Engineer (2009) from Pinar del Río University (UPR), Pinar del Río, Cuba". Currently he works as Assistant Professor in the Department of Telecommunication and Electronic Engineering in Pinar del Río University (UPR).

Jesus M. Corres received the MS degree in electrical engineering from the Public University of Navarra, Pamplona, Spain, in 1996 and the PhD degree from the Public University of Navarra, Pamplona, Spain in 2003. Currently he works as Associate Professor in the Department of Electrical and Electronic Engineering (UPNA). His main research is the development of fiber optic sensors using nanostructured materials for biomedical, environmental and safety applications. He is the author or co-author of more than 100 publications and serves as associate editor of IEEE Sensor Letters and Hindawi Journal of Sensors.

Ignacio R. Matias received the M.S. degree in Electrical and Electronic Engineering and his Ph.D. degree in Optical Fiber Sensors from the Polytechnic University of Madrid (UPM), Madrid, Spain, in 1992 and 1996, respectively. He became a Lecturer at the Public University of Navarra (Pamplona, Spain) in 1996, where presently he is a Permanent Professor. He has co-authored more than 300 chapter books, journals and conference papers related to optical fiber sensors and passive optical devices and systems. He is a Senior Editor of "IEEE Sensors Journal". He is an IEEE Senior member. He is a Topical Editor of the IEEE Sensors Journal 